\title{
A PROOF OF THE IDENTITY OF THE RIESZ INTEGRAL AND THE LEBESGUE INTEGRAL*
}

\author{
BY W. M. WHYBURN
}

1. Introduction. In Acta Mathematica, volume 42, pages 191-205, Friedrich Riesz developed a theory of integration independent of the theory of measure of point sets except in so far as sets of measure zero were involved. His theory yields an integral which he showed to have many of the properties of the Lebesgue integral and which he showed to exist and to be identical with the Lebesgue integral when this latter integral exists. Riesz' treatment yields a theory of measure which has the essential characteristics of the Lebesgue theory. Riesz does not seem to have proved that his development yields an integral which is identical with the Lebesgue integral $\dagger$ and that his notion of measure is the same as the ordinary notion. The present paper does show the entire identity of the Riesz integral and the Lebesgue integral. The terminology and notation of Riesz' paper are used.

Theorem. A necessary and sufficient condition that a bounded function on $X: a \leqq x \leqq b$ be measurable is that there exist a sequence of simple functions $\ddagger$ which approaches this function almost everywhere on $X$.

2. Proof of Necessity. $\S$ Let $f(x)$ be bounded and measurable on $X$ and let a method of subdivision of $X$ be chosen in such a way that the length of the longest subdivision approaches zero as the number of these subdivisions becomes infinite. $f(x)$ is Lebesgue integrable on $X$. Let $a=x_{0}<x_{1}<x_{2}<\cdots<x_{n}=b$ be the subdivision points at the $n$th stage and let

* Presented to the Society, April 11, 1931.

$\dagger$ At the bottom of page 199 of Riesz' paper one finds a statement which indicates that he suspected a close relationship between or even the identity of, the two integrals; but he makes no positive statement, and gives no proof.

$\ddagger$ Also called step functions or horizontal functions.

$\S$ This part of the theorem is well known. A proof is included here for completeness. 


$$
\phi_{n}(x)=\int_{x_{i-1}}^{x_{i}} \frac{f(t) d t}{x_{i}-x_{i-1}}
$$

on $x_{i-1}<x<x_{i},(i=1,2, \cdots, n)$. Since $F(x)=\int_{a}^{x}(t) d t$ is absolutely continuous on $X$, its derivative exists and is equal to $f(x)$ almost everywhere on $X$. Let $A$ be the point set of zero measure that is made up of all points of $X$ for which $F^{\prime}(x)$ fails to exist together with all subdivision points. Let $x=p$ be any point of $X-A$ and let the notation be chosen so that $x_{i}<p$ $<x_{i+1}$ for each $n$. We have

$$
\begin{aligned}
\phi_{n}(p)=\int_{x_{i}}^{x_{i+1}} \frac{f(x) d x}{x_{i+1}-x_{i}} & =\int_{x_{i}}^{p} \frac{f(x) d x}{p-x_{i}}+\left[\int_{p}^{x_{i+1}} \frac{f(x) d x}{x_{i+1}-p}\right. \\
& \left.-\int_{x_{i}}^{p} \frac{f(x) d x}{p-x_{i}}\right] \frac{x_{i+1}-p}{x_{i+1}-x_{i}} .
\end{aligned}
$$

Since $\left(x_{i+1}-p\right) /\left(x_{i+1}-x_{i}\right)<1$ and each of the terms in the bracket of (1) approaches $F^{\prime}(p)=f(p)$, it follows that the second term of the right hand side of equation (1) approaches zero when $n$ becomes infinite. The first term on the right hand side of (1) has $F^{\prime}(p)=f(p)$ for its limit as $n$ becomes infinite (since $x_{i} \rightarrow p$ ) and hence $\lim _{n \rightarrow \infty} \phi_{n}(p)=f(p)$. We have therefore shown that $\left\{\phi_{n}(x)\right\}$ approaches $f(x)$ almost everywhere on $X$. We also note that the area under $\phi_{n}(x)$ is $\int_{a}^{b} f(x) d x$ for each $n$.

3. Proof of Sufficiency. We give two proofs of this part of the theorem. Let $\left\{\phi_{n}(x)\right\}$ be a uniformly bounded sequence of simple functions approaching $f(x)$ almost everywhere on $X$ (such a sequence can be constructed from the set given in the theorem by substituting the bounds of $f(x)$ for portions of functions which exceed these bounds in numerical value). By Egeroff's theorem* $\left\{\phi_{n}(x)\right\}$ approaches $f(x)$ uniformly on $X$ except for a set of points of arbitrarily small outer measure. Let $M$ be any constant. We show that the sets on which $f(x)>M$, $f(x)<M$, and $f(x)=M$ are measurable. Let $\epsilon$ be an arbitrarily assigned positive number and let $N_{i}$ be chosen so that for all $n \geqq N_{i}$ and for all $x$ on $X$ except for points of a set of outer measure less than $\epsilon / 2^{i+1},\left|f(x)-\phi_{n}(x)\right|<1 / 2^{i}$. Let $G_{i}$ be the

* See Hobson, Functions of a Real Variable, 1926, vol. 2, p. 140. 
finite set of intervals on which $\phi_{N_{i}}(x)>M+1 / 2^{i}$. With the possible exception of a set of points of outer measure less than $\epsilon / 2^{i+1}, f(x)>M$ on $G_{i}$. The set $\sum G_{i}$ that is obtained when $i$ takes on all positive integral values may be replaced by an at most countably infinite set $g$ of non-overlapping intervals which has the same interior. Let $L$ be the sum of the lengths of the intervals of $g$ and note that $g$ contains all points of $X$ for which $f(x)>M$ except for points of the null set on which $\left\{\phi_{n}(x)\right\}$ does not converge to $f(x)$. The subset of $g$ on which $f(x) \leqq M$ is of outer measure less than $\sum_{1}^{\infty} \epsilon / 2^{\imath+1}<\epsilon$ and hence the inner measure of the set on which $f(x)>M$ differs from $L$ by less than $\epsilon$. The outer measure of the set on which $f(x)>M$ cannot exceed $L$ since $g$ is an admissible covering of this set. The set on which $f(x)>M$ is therefore measurable since its inner and outer measures differ by an arbitrary number $\epsilon$. Similar reasoning shows that the set on which $f(x)<M$ is measurable. The set on which $f(x)=M$ is measurable, since it is the difference between the measurable set $X$ and the sum of the two sets whose measurabilities have just been demonstrated. Hence $f(x)$ is measurable on $X$.

4. Second Proof of Sufficiency. We use the Baire classification of functions.* Let $A$ be the set of all points of $X$ at which any function of $\left\{\phi_{n}(x)\right\}$ is discontinuous and let $B$ be the set of points at which $\left\{\phi_{n}(x)\right\}$ does not approach $f(x)$. The set $C=A+B$ is of measure zero and the sets $X-A$ and $X-C$ are dense in themselves. For each $n, \phi_{n}(x)$ is of the first class $\dagger$ on $X-A$. Hence $f(x)$ is of the second class on $X-C$. It follows that $f(x)$ is measurable $\ddagger$ on $X-C$ and is therefore measurable on $X$.

The foregoing theorem shows that the class of bounded functions that are integrable in the Riesz sense is identical with the class of bounded functions that are integrable in the Lebesgue sense. Riesz' results show that the two integrals are equal when they both exist, and we have shown in this paper that when either exists, the other does. The complete equivalence of the two integrals for bounded functions is therefore established. In the presence of this equivalence one needs but to examine the

* See Carathéodory, Vorlesungen ueber reelle Funktionen, 1927, pp.393-413.

$\uparrow$ See Carathéodory, loc. cit., page 403, Satz 3.

¥ See Carathéodory, loc. cit., page 403, Satz 4. 
definitions of Riesz and Lebesgue for integrals of unbounded functions to see that the equivalence extends to such functions. We may therefore state that the class of functions summable in the Lebesgue sense is identical with the class of functions summable in the Riesz sense and the two theories lead to one and the same integral. As a corollary to this result we get the equivalence of the Riesz and Lebesgue theories of measure.

In connection with unbounded functions it might be observed that one could define the integral of such a function so that it would exist when either the Lebesgue integral or the improper Riemann integral exists.* Such a definition would completely remove the necessity of separate treatments of Lebesgue and Riemann integration as it, together with the results of the present paper, would permit both theories to be presented simultaneously from the Riesz point of view. The following two theorems emphasize this point of view.

$A$ necessary and sufficient condition that a bounded function be Lebesgue integrable on $X: a \leqq x \leqq b$ is that it be the limit function almost everyze here of a sequence $\left\{\phi_{n}(x)\right\}$ of simple functions.

The present paper establishes this theorem. Riesz $\uparrow$ proves the following second theorem.

$A$ necessary and sufficient condition tinat a bounded Lebesgue integrable function be Riemann integrable is that the sequence $\left\{\phi_{n}(x)\right\}$ approach the function uniformly $\ddagger$ almost everywhere on $X$.

The University of California at Los Angeles

* Since the present paper was written, I have used the Riesz point of view to define an integral of an unbounded function which has this property. This integral is unique and it may exist when neither the Lebesgue nor the Riemann integral exists. I have also established a necessary and sufficient condition (in terms of simple functions) that a function (which may be unbounded) be summable in the Lebesgue sense. It is my intention to publish these results in the near future.

$\dagger$ Loc. cit., p. 204.

$\ddagger$ Riesz, loc. cit., p. 204, defines uniform approach almost everywhere on $X$ to mean uniform approach in the neighborhoods of all points of $X$ with the possible exception of a set of measure zero. 